\title{
ANFIS Approach for Navigation of Mobile Robots
}

\author{
Singh Mukesh Kumar \\ Parhi Dayal R. \\ Pothal Jayanta Kumar \\ Department of Mechanical Engineering Department of Mechanical Engineering Department of Mechanical Engineering \\ Government Engineering College Bilaspur National Institute of Technology Rourkela National Institute of Technology Rourkela \\ Chhattisgarh, India. \\ e-mail:mukesh3003@gmail.com \\ Orissa, India \\ e-mail:dayalparhi@yahoo.com \\ Orissa, India \\ e-mail: jayantapothal@yahoo.co.in
}

\begin{abstract}
This paper, discusses about navigation control of mobile robot using adaptive neuro-fuzzy inference system (ANFIS) in a real word dynamic environment. In the ANFIS controller after the input layer there is a fuzzy layer and rest of the layers are neural network layers. The adaptive neuro-fuzzy hybrid system combines the advantages of fuzzy logic system, which deal with explicit knowledge that can be explained and understood, and neural network, which deal with implicit knowledge, which can be acquired by learning. The inputs to fuzzy logic layer are front obstacle distance, left obstacle distance, right obstacle distance and target steering. A learning algorithm based on neural network technique has been developed to tune the parameters of fuzzy membership functions, which smooth the trajectory generated by the fuzzy logic system. Using the developed ANFIS controller, the mobile robots are able to avoid static and dynamic obstacles, and reach the target successfully in cluttered environments. The experimental results agree well with the simulation results, proves the authenticity of the theory developed.
\end{abstract}

Keywords-Robotsbehavior; ANFIS; dynamic environments.

\section{INTRODUCTION}

The current robot navigation systems require controllers able to solve complex problems under very uncertain and dynamic environmental situations. Presently, the ANFIS approach is becoming one of the major areas of interest because it gets the benefits of neural networks as well as of fuzzy logic systems and it removes the individual disadvantages by combining them on the common features [13]. The artificial neural network has injected a new driving force into the fuzzy literature. The artificial neural network can be used as a universal learning paradigm for any smooth parameterized models, including fuzzy inference system [4].

Traditional robot control methods rely upon strong mathematical modeling, analysis, and synthesis. The approaches, proposed to date, are suitable for control of mobile robots which operate in unknown environments and perform desired tasks that require motion in dynamic environment. However, operations in unstructured environments, such as in remote planets and hazardous waste sites, require robots to perform more complex tasks without an adequate analytical model [5]. In recent years many researchers and engineers have tried to solve navigation problem of mobile robot system [6-8]. Fuzzy systems have the ability to make use of knowledge expressed in the form of linguistic rules, thus they offer the possibility of implementing expert human knowledge and experience [9-12]. The main drawback of fuzzy controller is the lack of a systematic methodology for their design. Usually, tuning parameters of membership functions is a time consuming task. Neural network learning techniques can automate this process, significantly reducing development time, and resulting in better performance. The merger of neural networks and fuzzy logic led to the creation of neuro-fuzzy controllers which are currently one of the most popular research fields [10]. For real time autonomous navigation, the robot should be capable of sensing its environment, interpreting the sensed information to obtain the knowledge of its position and the environment, planning a real-time route from an initial position to a target with obstacle avoidance, and controlling the robot direction and velocity to reach the target [13]. $\mathrm{Ng}$ et al. [14] have proposed a neural integrated fuzzy controller, which integrates the fuzzy logic representation of human knowledge with the learning capability of neural networks, to slove nonlinear dynamic control problems. Pham et al. [15] have focused on the development of intelligent multi-agent robot teams that are capable of acting autonomously and of collaborating in a dynamic environment to achieve team objectives. They have also proposed a neuro-fuzzy based adaptive action selection architecture that enables team of robot agents to achieve adaptive cooperative control to perform cooperative tasks, dynamic target tracking and box pushing. The problem of autonomous navigation applied to mobile robots has well defined as a search process within a navigation environment containing obstacles and targets by Crestani et al. [16]. They have proposed a fuzzy-neural-network-based controller that considers the direction and the velocity of navigation as controllable terms. Rutkowski et al. [17] have derived a flexible neuro-fuzzy inference system. Their approach introduced more flexibility to the structure and design of neurofuzzy systems. Neuro-fuzzy controllers for sensor-based mobile robot navigation have well discussed by Hui et al.[18] and Rusu et al. [19]. An adaptive neuro-fuzzy inference system implemented in a robotic vehicle navigation by Garbi et al.[20].

In this paper ANFIS approach has been analyzed for real time navigation of mobile robots, in the present work, to determine time-optimal, collision-free path of mobile robot navigating in unknown and dynamic environment. Numerical examples are presented to demonstrate the validity of the approach. The simulation results are compared with the results from other methods [7, 10, and 11]. Also experimental results are verified with simulation results to demonstrate the superiority of the proposed methodology.

This paper is organized into five sections following the introduction, the ANFIS architecture has been discussed in section II. The simulation results are presented in section III 
and experimental results are analyzed in section IV. Finally conclusions are given in section $\mathrm{V}$.

\section{ANFIS APPROACH}

The adaptive neuro fuzzy inference system (ANFIS) is an integrated system of artificial neural network (ANN) and fuzzy inference system (FIS). The ANFIS analysed here is a first order Takagi Sugeno Fuzzy Model [4], [14]. In the current analysis there are four inputs: Front obstacle distance $\left(\mathrm{x}_{1}\right)$, Right obstacle distance $\left(\mathrm{x}_{2}\right)$, Left obstacle distance $\left(\mathrm{x}_{3}\right)$ and Target angle $\left(\mathrm{x}_{4}\right)$ and the output is Steering angle. The if-then rules for the ANFIS architecture are defined as follows;

Rule: IF $x_{1}$ is $A_{j} ; x 2$ is $B_{k} ; x_{3}$ is $C_{m}$ and $x_{4}$ is $D_{n}$

THEN $F_{i}=p_{i} x_{1}+r_{i} x_{2}+s_{i} x_{3}+t_{i} x_{4}$

Where

$\mathrm{F}_{\mathrm{i}}=\mathrm{p}_{\mathrm{i}} \mathrm{x}_{1}+\mathrm{r}_{\mathrm{i}} \mathrm{x}_{2}+\mathrm{s}_{\mathrm{i}} \mathrm{x}_{3}+\mathrm{t}_{\mathrm{i}} \mathrm{x}_{4}+\mathrm{u}_{\mathrm{i}}$ for steering angle

$\mathrm{J}=1$ to $\mathrm{q}_{1} ; \mathrm{k}=1$ to $\mathrm{q}_{2} ; \mathrm{m}=1$ to $\mathrm{q}_{3} ; \mathrm{n}=1$ to $\mathrm{q}_{4}$ and $\mathrm{i}=1$ to $\mathrm{q}_{1} \cdot \mathrm{q}_{2} . \mathrm{q}_{3} . \mathrm{q}_{4}$

A, B, C and D are the fuzzy membership sets defined for the input variables $\mathrm{x}_{1}, \mathrm{x}_{2}, \mathrm{x}_{3}$ and $\mathrm{x}_{4} . \mathrm{q}_{1}, \mathrm{q}_{2}, \mathrm{q}_{3}$ and $\mathrm{q}_{4}$, are the number of member ship functions for the fuzzy systems of the inputs $\mathrm{x}_{1}, \mathrm{x}_{2}, \mathrm{x}_{3}$ and $\mathrm{x}_{4}$ respectively. $\mathrm{f}_{\mathrm{i}}$ is the linear consequent functions defined in terms of the inputs $\left(x_{1}, x_{2}, x_{3}\right.$ and $\left.x_{4}\right) . q_{i}$, $r_{i}, s_{i}, t_{i}$ and ui are the consequent parameters of the ANFIS fuzzy model. In the ANFIS model, nodes of the same layer have similar functions. The output signals from the nodes of the previous layer are the input signals for the current layer. The output obtained with the help of the node function will be the input signals for the subsequent layer as shown in Fig. 1.

Layer 1: The input layer receives signal from arrays of sensors $\mathrm{x}_{1}, \mathrm{x}_{2}, \mathrm{x}_{3}$ and $\mathrm{x}_{4}$ which defines the static as well as moving obstacles and target poisons from the target tracker robot.

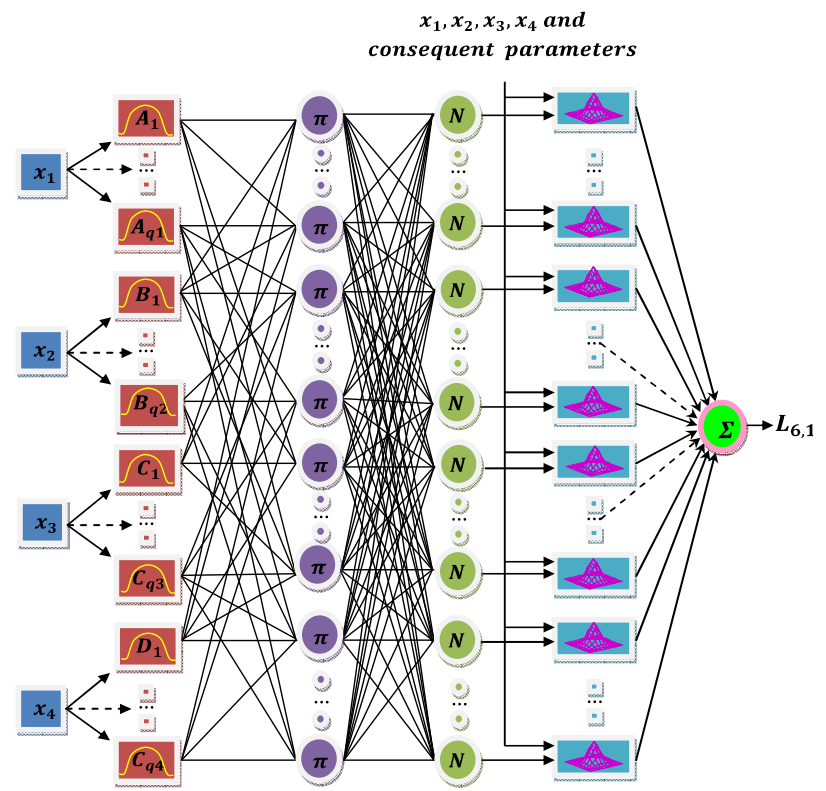

Figure 1. Six layer ANFIS for robot navigation.
Layer 2: Every node in this layer is an adaptive node (square node) with a particular fuzzy membership function (node function) specifying the degrees to which the inputs satisfy the quantifier. For four inputs the outputs from nodes are given as follows;

$\mathrm{L}_{2 \mathrm{~g}}=\mathrm{U}_{\mathrm{ag}}(\mathrm{x})$ for $\mathrm{g}=1, \ldots, \mathrm{q}_{1}$

(for input $\mathrm{x}_{1}$ )

$\mathrm{L}_{2 \mathrm{~g}}=\mathrm{U}_{\mathrm{Bg}}(\mathrm{x})$ for $\mathrm{g}=\mathrm{q}_{1}+1, \ldots, \mathrm{q}_{1}+\mathrm{q}_{2}$

(for input $\mathrm{x}_{2}$ )

$\mathrm{L}_{2 \mathrm{~g}}=\mathrm{U}_{\mathrm{Cg}}(\mathrm{x})$ for $\mathrm{g}=\mathrm{q}_{1}+\mathrm{q}_{2}+1, \ldots, \mathrm{q}_{1}+\mathrm{q}_{2}+\mathrm{q}_{3}$

(for input $\mathrm{x}_{3}$ )

$\mathrm{L}_{2 \mathrm{~g}}=\mathrm{U}_{\mathrm{Dg}}(\mathrm{x})$ for $\mathrm{g}=\mathrm{q}_{1}+\mathrm{q}_{2}+\mathrm{q}_{3}+1, . ., \mathrm{q}_{1}+\mathrm{q}_{2}+\mathrm{q}_{3}+\mathrm{q}_{4}$ (for input $\mathrm{x}_{4}$ )

Here the membership function for A, B, C and D considered are the bell shaped function and are defined as follows;

$$
\begin{aligned}
& ; \mathrm{g}=1 \text { to } \mathrm{q}_{1} \\
& \mu_{A g}(x)=\frac{1}{1+\left\{\left(\frac{x-c_{g}}{a_{g}}\right)^{2}\right\}^{b_{g}}} \\
& \mu_{B g}(x)=\frac{1}{1+\left\{\left(\frac{x-c_{g}}{a_{g}}\right)^{2}\right\}^{b_{g}}} ; \mathrm{g}=\mathrm{q}_{1}+1 \text { to } \mathrm{q}_{1}+\mathrm{q}_{2} \\
& \mu_{B g}(x)=\frac{1}{1+\left\{\left(\frac{x-c_{g}}{a_{g}}\right)^{2}\right\}^{b_{g}}} ; \mathrm{g}=\mathrm{q}_{1}+\mathrm{q}_{2}+1 \text { to } \mathrm{q}_{1}+\mathrm{q}_{2}+\mathrm{q}_{3}(3) \\
& \mu_{B g}(x)=\frac{1}{1+\left\{\left(\frac{x-c_{g}}{a_{g}}\right)^{2}\right\}^{b_{g}}} \\
& \mathrm{~g}=\mathrm{q}_{1}+\mathrm{q}_{2}+\mathrm{q}_{3}+1 \text { to } \mathrm{q}_{1}+\mathrm{q}_{2}+\mathrm{q}_{3}+\mathrm{q}_{4}
\end{aligned}
$$

Where $a_{g}, b_{g}$ and $c_{g}$ are the parameters for the fuzzy membership function. The ball-shaped function changes its pattern as per the change of the parameters. This change will give the various contour of bell shaped function as needed in accord with the data set for the problem considered.

Layer 3: Every node in this layer is a fixed node (circular) labeled as ' $\pi$ '. The output denoted by $L_{2 i}$ the output is the product of all incoming signal.

$\mathrm{L}_{3 \mathrm{i}}=\mathrm{W}_{\mathrm{i}}=\mathrm{U}_{\mathrm{ag}}(\mathrm{x}), \mathrm{U}_{\mathrm{Bg}}(\mathrm{x}), \mathrm{U}_{\mathrm{Cg}}(\mathrm{x}), \mathrm{U}_{\mathrm{Dg}}(\mathrm{x})$;

For $\mathrm{i}=1, \ldots, \mathrm{q}_{1} \cdot \mathrm{q}_{2}, \mathrm{q}_{3}, \mathrm{q}_{4}$ and $\mathrm{g}=1, \ldots, \mathrm{q}_{1}+\mathrm{q}_{2}+\mathrm{q}_{3}+\mathrm{q}_{4}$

The output of each node of the second layer represents the firing strength (degree of fulfillment) of the associated rule. The $\mathrm{T}$-nom operator algebraic product $\left\{\mathrm{T}_{\mathrm{ap}}(\mathrm{a}, \mathrm{b})=\mathrm{ab}\right\}$, has been used to obtain the firing strength $\left(\mathrm{W}_{\mathrm{i}}\right)$.

Layer 4: Every node in this layer is a fixed node (circular) labeled as "N". The output of the $i^{\text {th }}$ node is calculated by taking the ratio of firing strength of $i^{\text {th }}$ rule $\left(\mathrm{W}_{\mathrm{i}}\right)$ to the sum of all rules' firing strength. 


$$
L_{4 i}=\bar{W}_{i} f_{i}=\frac{W_{i}}{\sum_{r-1} W_{r}}
$$

This output gives a normalized firing strength.

Layer 5: Every node in this layer is an adaptive node (square node) with a node function.

$$
L_{4 i}=\bar{W}_{i} f_{i}=\bar{W}_{i}\left(p_{i} x_{1}+r_{i} x_{2}+s_{i} x_{3}+t_{i} x_{4}+u_{i}\right)
$$

Where $\bar{W}_{i}$ is a normalized firing strength form (output) from layer 3 and $\left\{\mathrm{p}_{\mathrm{i}}, \mathrm{r}_{\mathrm{i}}, \mathrm{s}_{\mathrm{i}}, \mathrm{t}_{\mathrm{i}}, \mathrm{u}_{\mathrm{i}}\right\}$ is the parameter set for steering angle. Parameters in this layer are referred to as consequent parameters.

Layer 6: The single node in this layer is a fixed node (circular) labeled as " $\Sigma$ ", which computes the overall output as the summation of all incoming signals.

$$
L_{6 i}=\sum_{r-1}^{r=q_{1} \cdot q_{2} \cdot q_{3} \cdot q_{4}} \bar{W}_{i} f_{i}=\frac{\sum_{i=1}^{i=q_{1} \cdot q_{2} \cdot q_{3} \cdot q_{4}} W_{i} f_{i}}{\sum_{i=1} W_{i}}
$$

In the current developed ANFIS structure there are six dimensional space partitions and has ' $\mathrm{q}_{1} \cdot \mathrm{q}_{2} \cdot \mathrm{q}_{3} \cdot \mathrm{q}_{4}$ ' regions. Each region is governed by a fuzzy if then rule. The first layer is the input layer. The second layer (consists of premise or antecedent parameters) of the ANFIS and is dedicated to fuzzy sub space. The parameters of the fifth layer are referred as consequent parameters and are used to optimize the network. During the forward pass of the hybrid learning algorithm node outputs go forward till layer five and the consequent parameters are identified by least square method. In the backward pass, error signals propagate backwards and the premise parameters are updated by a gradient descent method.

\section{SIMULATION RESULTS AND DISCUSSION}

The simulation results are obtained by using ROBNAV software which has been developed in the laboratory using $\mathrm{C}++$ [21]. Fig. 2 shows a typical screen of the software. To demonstrate the effectiveness and the robustness of the proposed method, simulation results on mobile robot navigation in various environments are exhibited.

The obstacle avoidance behavior is activated when the reading from any sensors are less than the minimum threshold values. This is how the robot determines if an object is close enough for a collision. When an object is detected too close to the robot, it avoids a collision by moving away from it in the opposite direction. Collision avoidance has the highest priority and therefore, it can override other behaviors, in this case, its main reactive behavior is decelerating for static as well as dynamic obstacle avoidance as shown in Fig. 3.

Another special condition appears as the mobile robot detects an obstacle in the front while the target tracking control mode is on operation. In this case, the fixed wall following behavior should be performed first, that is, the mobile robot must rotate clockwise or counterclockwise such that it can align and move along the wall (see Fig.4). Any obstacle- avoidance behavior except wall following behavior would make the robot divert from its goal position. When the acquired information from the sensors shows that there are no obstacles around robot, its main reactive behavior is target steer. ANFIS mainly adjusts robots motion direction and quickly moves it towards the target if there are no obstacles around the robot as shown in Fig. 3. In the proposed control strategy, reactive behaviors are formulated and trained by ANFIS.

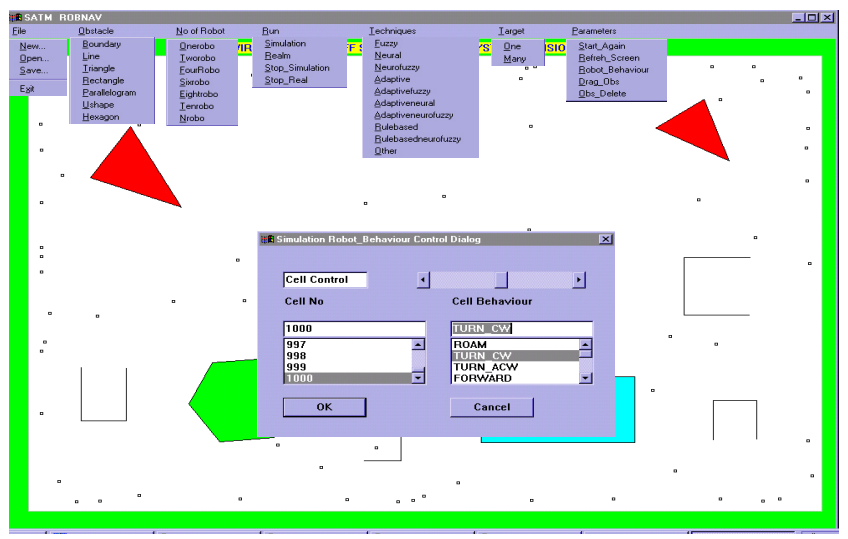

Figure 2. Robot navigation software package (ROBNAV).

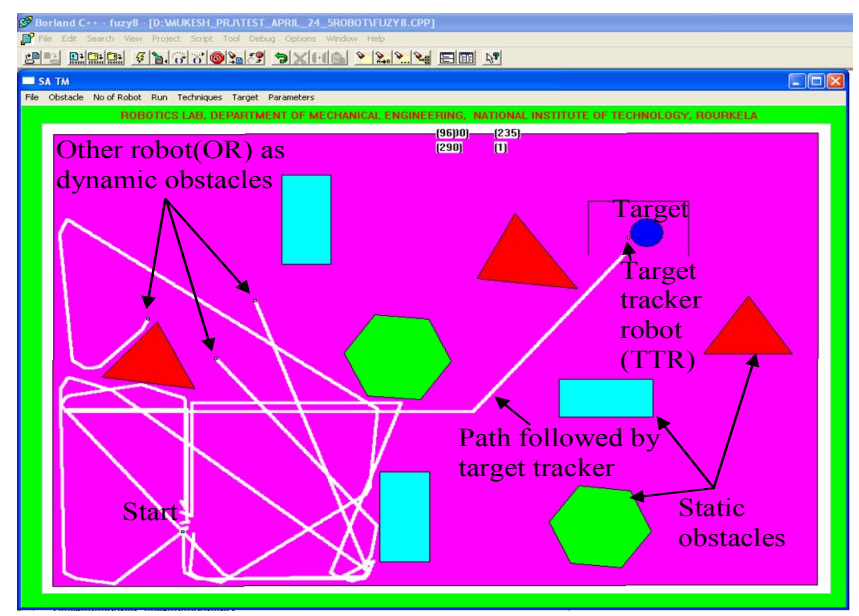

Figure 3. Static as well as dynamic obstacle avoidance behabior.

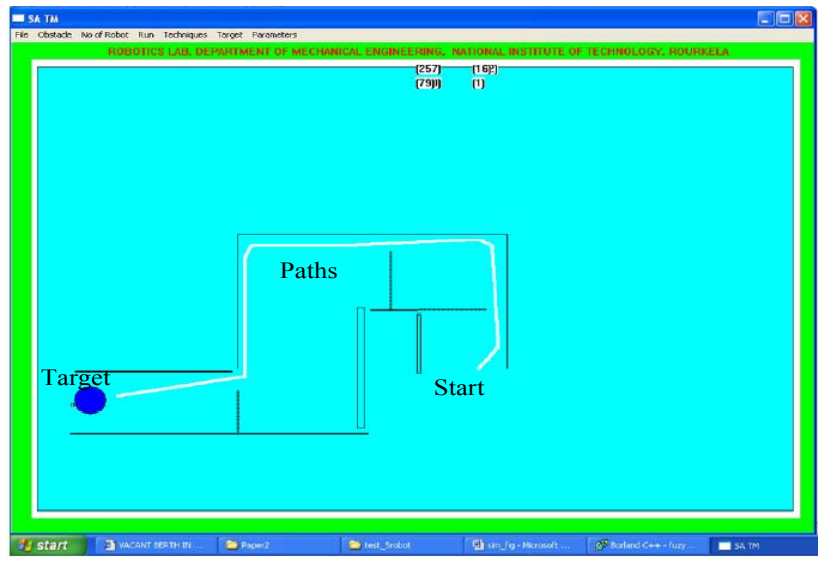

Figure 4. Escaping from dead end obstacle by wall following behavior. 
The results from the proposed method for real time navigation of mobile robot have been compared with the result from fuzzy controller by Pradhan et al.[10] shown in Fig. 5(a), neural controller by Parhi et al.[8] shown in Fig. 5(b). During analysis, it has been found that the developed ANFIS has better performance than the fuzzy as well as neural controller in terms of path and time optimization as shown in Fig. 5(c). A comparison has also been done between the results obtained by Abdessemed et al.[10] for two environments and the current developed ANFIS method [Fig. 6(a) and Fig. 6(b)]. They show a good agreement.

\section{EXPERIMENTAL RESULTS}

Experimental results are found using Khepra-III mobile robot. The chassis of the robot measures $130 \times 70 \mathrm{~mm}\left(\mathrm{D} \times_{\mathrm{H}}\right)$, weight $690 \mathrm{~g}$, pay load $2000 \mathrm{~g}$ approx. fitted with DsPIC $30 \mathrm{~F} 5011$ at $60 \mathrm{MHz}$ processor with $4 \mathrm{~KB}$ on DsPIC and $64 \mathrm{MB}$ KoreBot RAM. It is equipped with $2 \mathrm{DC}$ brushed servo motors with incremental encoders and 9 Infra-red proximity and ambient light sensors 2 Infra-red ground proximity sensors for line following applications 5 Ultrasonic sensors with range 0.24 meters. The speed range is $0-0.5 \mathrm{~m} / \mathrm{s}$, Lithium-Polymer battery pack $(1400 \mathrm{mAh})$ is used for powering the robot as shown in Fig. 7. The assumptions about the mechanical structure and the motion of a mobile robot to which our proposed method is applied are mobile robot moves on lab specified floor area and the wheel of a mobile robot rolls on the floor without any translational slip.

The experimental paths followed by mobile robots to reach the target are traced. From the ANFIS (inputs: left, front, right obstacle distances and target angle) after learning, training and testing, robots gets the new steering angles. The paths traced by the obstacle robots (OR) and target tracker robot (TTR) are marked on the floor by a pen as they move. The OR1, OR2, OR3, OR4 (Khepra-II) are term as moving obstacle and TTR (Khepra-III) is terms as the target tracker. The paths followed by OR and TTR have been shown in Fig. 7. The results obtained from experimental setup are more close to results obtained from simulation mode which validate the proposed method.

From these figures, it can be seen that the robots can indeed avoid obstacles and reach the targets. To verify the feasibility of proposed method Table 1 shows a comparison between fuzzy, neural and ANFIS controller.

TABLE I. COMPARISION WITH VARIOUS SCENARIO

\begin{tabular}{|l|c|c|c|}
\hline S.N. & $\begin{array}{c}\text { Simulation results of different } \\
\text { methods }\end{array}$ & $\begin{array}{c}\text { Length of } \\
\text { path (M) }\end{array}$ & Time (s) \\
\hline 1. & $\begin{array}{c}\text { Pradhan et al. [10] with fuzzy } \\
\text { controller, Fig. 5(a). }\end{array}$ & 13.8 & 14.63 \\
\hline 2. & $\begin{array}{c}\text { Parhi et al. [8] with neural controller, } \\
\text { Fig. 5(b). }\end{array}$ & 12.2 & 12.93 \\
\hline 3. & With purposed ANFIS, Fig. 5(e). & 6.6 & 7.02 \\
\hline
\end{tabular}

These robotics behaviors are verified in simulation and experimental mode. Table 2 shows the times taken by the robots in simulations and in the experimental tests scenario during target finding. It is observed that the robots are able to reach the targets efficiently during simulation and experiment.
It is found that the navigation of mobile robot with purposed ANFIS method has better performance than the fuzzy as well as neural controller in terms of positioning accuracy and collision avoidance.

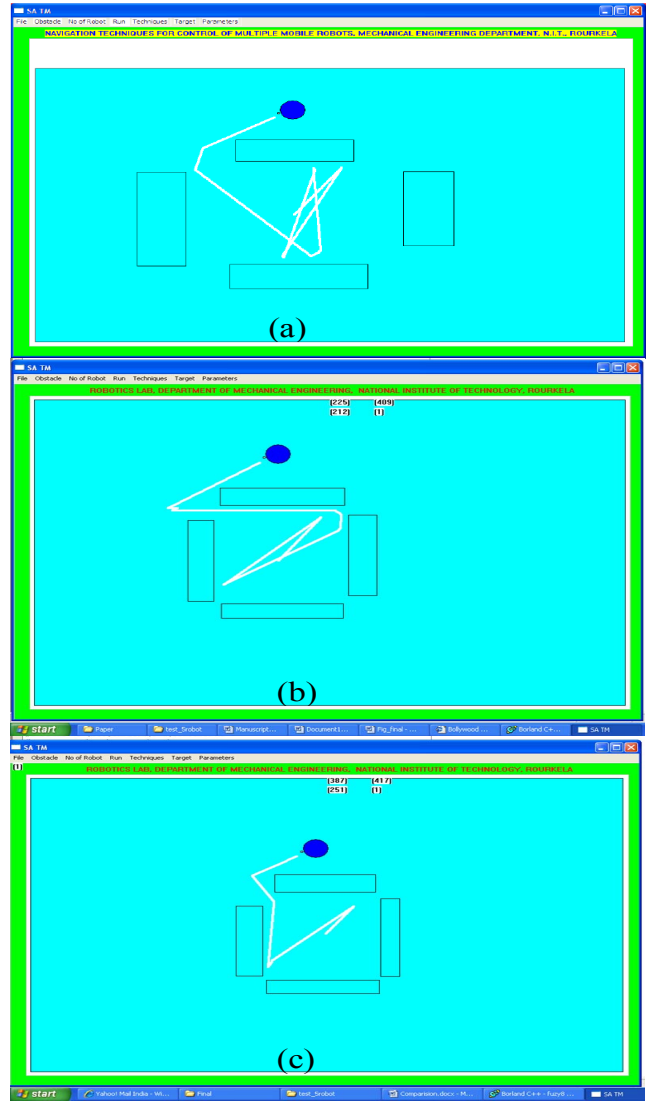

Figure 5. Comparision of results (a) Navigational path using fuzzy controller by Pradhan et al.[10] (b) Navigational path using neural controller by Parhi et al.[8] (c) Navigational path by perposed ANFIS appoach.

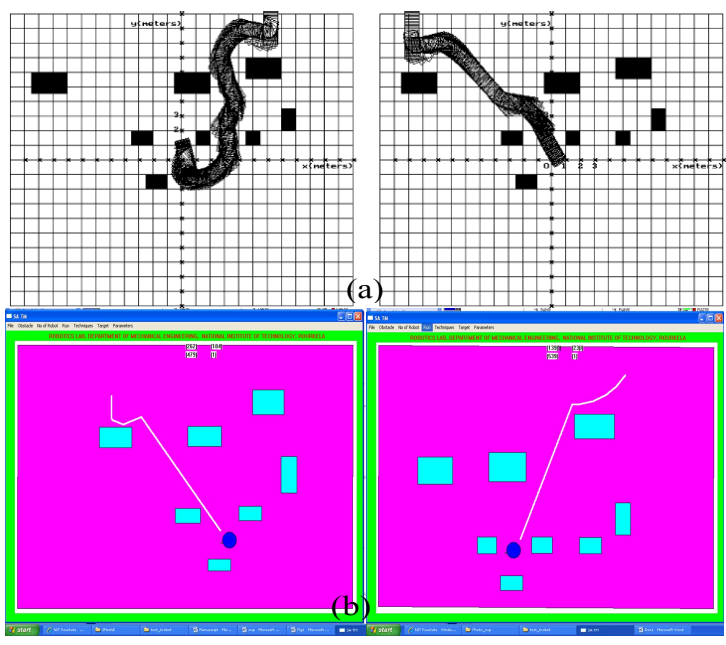

Figure 6. Comparision of results (a) Navigational path during vehicle controlled motion with a cluttered obstacle environment from two different starting points by Abdessemed et al.[11] (b) Navigational path by proposed ANFIS approach. 


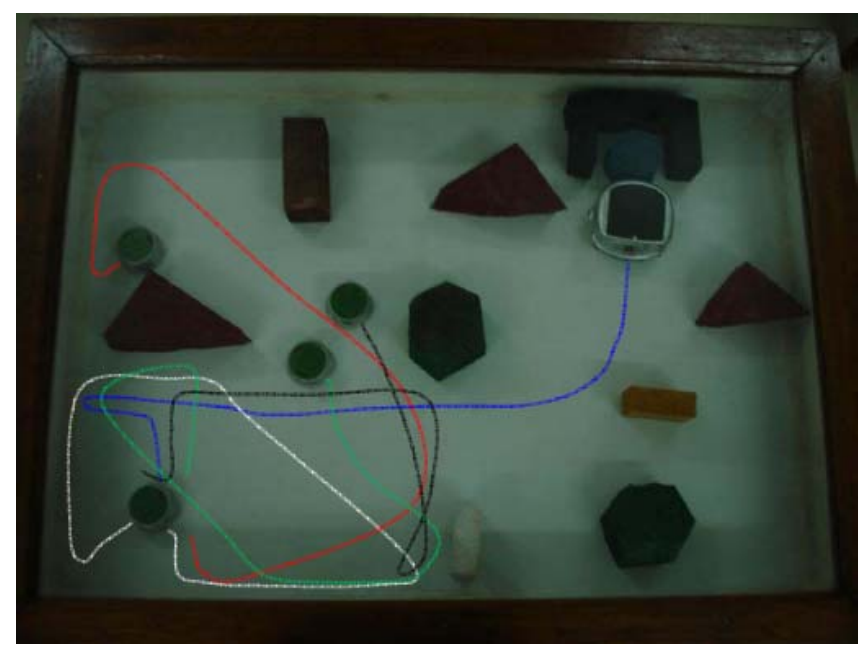

Figure 7. Experimental result duiring navigation of mobile robots.

TABLE II. OBSERVATIONS DURING SIMULATIONS AND EXPERIMENT

\begin{tabular}{|c|c|c|c|c|}
\hline $\begin{array}{c}\text { S. } \\
\text { No }\end{array}$ & Observations & $\begin{array}{c}\text { Simulation } \\
\text { environme } \\
\text { nt }\end{array}$ & $\begin{array}{c}\text { Experimental } \\
\text { environment }\end{array}$ & $\begin{array}{c}\text { Deviation of } \\
\text { results } \\
\text { (simulation v/s } \\
\text { experiment) }\end{array}$ \\
\hline 1. & $\begin{array}{c}\text { Length of } \\
\text { path ( meter) }\end{array}$ & 15.4 & 16.2 & $05.19 \%$ \\
\hline 2. & $\begin{array}{c}\text { Time taken } \\
\text { (sec.) }\end{array}$ & 16.37 & 18.63 & $08.49 \%$ \\
\hline
\end{tabular}

\section{CONCLUSIONS}

The developed ANFIS controller has been used for navigation control of multiple mobile robots both in simulation and experimental mode. ROBNAV software has been developed to the handle the navigation control of mobile robot using ANFIS controller. The methodology adopted here has got the following feature:

1. Using the ANFIS controller mobile robot able to avoid static as well dynamic obstacle in a cluttered environment.

2. The kinematic analysis of the mobile robot helps in giving the proper steering angle for the robot during the navigation.

3. The robots are able to move the surroundings using the embedded infrared sensors.

4. Comparisons with the different technique with the currently developed technique and the agreement in result show the effectiveness of the proposed technique.

In the future more robust hybrid technique may be developed for better navigation control.

\section{REFERENCES}

[1] S.K. Pradhan, D.R. Parhi and A.K.Panda, Neuro-fuzzy technique for navigation of multiple mobile robots, Fuzzy Optim. Decis. Making, 5, pp. 255-288, 2006.

[2] S.K. Pradhan, D.R. Parhi and A.K.Panda, Motion control and navigation of multiple mobile robots for obstacle avoidance and target seeking: a neuro-fuzzy rule-based technique, J. of Sys. and Cont. Engineering, vol. 223 no.2, pp. 275-288, 2009.

[3] S.K. Pradhan, D.R. Parhi and A.K.Panda, Navigation of multiple mobile robots using rule-based neuro-fuzzy technique, Int. J. of Comput. Intell., vol.3 no. 2, pp. 142-152, 2006.

[4] J.S.R. Jang, ANFIS: Adaptive-network-based fuzzy inference systems., IEEE Trans. on Syst. Man, and Cybern., vol.23 no.5, pp. 665-685, 1993.

[5] T.M.R. Akbarzadeh, K. Kumbla, E. Tunstel, and M. Jamshidi, . Soft comuting for autonomous robotic systems, Computers and Electrical Engineering, vol. 26, pp. 5-32, 2000.

[6] D.R. Parhi, S.K. Pradhan, A.K. Panda, and R.K. Behra, The stable and precise motion control for multiple mobile robots, Applied Soft Comput., vol.9 no. 2, pp.477-487, 2009.

[7] M.K.Singh and D.R. Parhi, Intelligent neuro-controller for navigation of mobile robot. In the International Conference on Comput. Intell. (ICAC3-09), Mumbai, India, January 2009, pp. 123-128.

[8] D.R. Parhi and M.K. Singh, Real time navigational control of mobile robots using artificial neural network, J. of Mech. Engineering part C, vol. 223no.7, pp.1713-1725, 2009.

[9] D.R. Parhi and M.K. Singh, Intelligent fuzzy interface technique for controller of mobile robot. J. of Mech. Engineering part C, vol. 222 no.11, pp. 2281-2292, 2008.

[10] S.K. Pradhan, D.R. Parhi and A.K.Panda, Fuzzy logic techniques for navigation of several mobile robots. Appl. Soft Comput., vol. 9, pp. 290 304, 2009

[11] F. Abdessemed, K. Benmahammedb, E. Monacelli, A fuzzy-based reactive controller for a non-holonomic mobile robot, Robot. and Auton. Sys., vol.47 pp.31-46, 2004.

[12] J. Godjevac and N. Steele, Neuro-fuzzy control of a mobile robot, Neurocomp., vol.28 pp. 127-143, 1999.

[13] O.R.E. Motlagh, T. Hong and S.N. Ismail, Development of a new minimum avoidance system for a behavior-based mobile robot, Fuzzy set and sys., vol.160 issue13 pp.1929-1946, 2008.

[14] K.C. Ng, and M.M. Trivedi, A Neuro-fuzzy controller for mobile robot navigation and multirobot convoying, IEEE Trans. on Sys., man, and cyber., part b, vol.28 no.6, pp.829-840, 1998.

[15] D.T.Pham,M.H. Awadalla, and E.E. Eldukhri, Fuzzy and neuro-fuzzy based co-operative mobile robots, In the 28th IEEE International Confrence on Industrial Electronics Society, Sevilla, Spain, November 2002, vol. 4 pp. 2962- 2967.

[16] P. Crestani, R. J. Fernando, and Z.A. Von, Hierarchical neuro-fuzzy approach to autonomous navigation. In the International Joint Conference on Neural Networks, Honolulu, HI, USA, 2002 pp. 23392344.

[17] L. Rutkowski and K. Cpalka, Flexible neuro-fuzzy systems, IEEE Trans. on neural networks, vol.14 no.3, pp. 554-574, 2003.

[18] N.B. Hui, V. Mahendar and D.K. Pratihar, Time-optimal, collision-free navigation of a car-like mobile robot using neuro-fuzzy approaches. Fuzzy Sets and Sys.,vol.157, pp. 2171-2204, 2006.

[19] P. Rusu, E.M.. Petriu, T.E.. Whalen,A. Cornell, and H.J.W. Spoelder, Behavior-based neuro-fuzzy controller for mobile robot navigation, IEEE Trans. on Instrument. and Measur., vol.52 no.4, pp. 1335-1340, 2003.

[20] G.P.Garbi, V. Orlando, G. Rosado and F.J.Grandinetti, Multivalued adaptive neuro-fuzzy controller for robot vehicle., In the International Confrense on Inteligence. System and knowledge Engineering, ISKE07, Chengdu, China, October 2007.

[21] D.R.Parhi, Navigation of multiple mobile robots in an unknown environment, Doctoral Thesis, Cardiff School of Engineering, University of Wales, UK, 2000. 